\section{A computerized cognitive laboratory: A software package for the cognitive psychology laboratory class}

\section{DAVID J. PITTENGER and BRUCE K. BRITTON University of Georgia, Athens, Georgia}

The package of programs described here allows a teacher/researcher to demonstrate several important concepts in the area of experimental cognitive psychology. These programs may be used either for an in-class demonstration of a particular concept or for a laboratory course that requires the student to collect and analyze data germane to the topic area being studied. There are similar packages available, such as the one described by Simpson and Burgess (1984); the present package, however, includes a broader range of demonstrations that can be used with most Commodore computers.

In general, all of the programs replicate an experiment or a standard procedure that has been used to examine a specific cognitive process. Indeed, when appropriate, the procedures of a well-known published experiment have been reproduced. All programs have been written in the BASIC language for the Commodore computer systems and will operate on both the CBM/PET series and the Commodore 64 system. The exceptions to this are the programs requiring color, which will operate only on the Commodore 64. The programs can also be copied and, with moderate modifications, be made compatible with other microcomputer systems (see Brain, Oviate, Paquin, \& Stone, 1981, for information on the translation process within the BASIC dialects for the Commodore, Apple II, and TRS- 80 systems). For experiments in which reaction times are measured, the system's internal " jiffy" " clock is used. This clock is accurate to within $1 / 60$ of a second, and all timed functions are reported to the nearest $1 / 100$ of a second.

Each program requires a minimum of instruction of the student and is constructed to decrease the chance of the program's being interrupted by inadvertent responses. The entire package is menu driven. That is, once the menu program is loaded into the computer, all demonstrations are loaded and run through program language. For the most part, the students are required only to use the " $<$ " and " $>$ " keys to register their responses. Each program offers brief instructions for the type of response required and has appropriate subroutines to detect incorrect responses. Programs that require students to use the al-

Funding for the software package was provided through a grant awarded to the second author by the Office of Instructional Development, University of Georgia, Athens, GA. All correspondence should be addressed to David J. Pittenger, Department of Psychology, University of Georgia, Athens, GA 30602. phabetic keys do not require fast entry of data, and, if necessary, typographical errors can be corrected easily. Typically, the demonstrations can be completed within $20 \mathrm{~min}$. At the conclusion of each demonstration, the data collected are presented, in tabular form, on the screen of the computer. A hard copy of the data may also be made if a printer is available.

\section{Description of Programs}

Free recall and recognition. This series of programs demonstrates the effects of two different types of memory task on the amount of material that can be retrieved from memory. In both demonstrations, a list of 20 words is selected randomly from a bank of words stored in the program, and each word is presented serially for $2 \mathrm{sec}$. After the list is presented, the subject is either engaged in a distracting task (determining whether a stimulus number is greater than or less than 500 ) before being tested or is tested immediately on the amount of the material remembered. During the second phase, the alternate condition (immediate recall/recognition, or masking) is presented. During this phase, a randomly generated list of different words is presented in the same manner and is followed by the appropriate task.

In the free-recall experiment, the subjects are required to write on a piece of paper the words that they remembered. This list is then compared with the memorized list, and the student records in his/her notes the number of words remembered correctly. For the recognition task, the subject is presented with a forced-choice test in which a word from the list and a nonlist word are presented. The student responds by pressing either the " $<$ " or the " $>$ " key to indicate which word was memorized. At the conclusion of this demonstration, the number of words identified correctly is presented for each delay condition. In both programs, the serial positions of the words are presented so that sequence effects may be analyzed.

The word bank for both programs contains 100 different words stored as DATA statements. If the user desires, the bank of words can be altered by rewriting the DATA statements and then resaving the program.

Stroop test. The effects of competing sensory information (see, e.g., Gordon, 1974) can be demonstrated effectively with this program (which operates only on the Commodore 64). There are three phases within the program. During the first phase, 20 color adjectives are flashed on the computer screen. The background is black, and the words are printed in white. Each word is presented and remains on until the subject has identified the word. In the second phase, 20 colored rectangles of approximately the same size as the adjectives are presented. Again, the sequence progresses as the subject identifies the color of the figure. In the third phase, 20 color adjectives are presented in competing colors, and the subject must identify the word that is presented. In each of the 
three phases, a correct response is registered by pressing any key on the keyboard. Given the nature of this task, it is suggested that the experiment be conducted with a laboratory assistant who will determine when the correct response has been made (the order of the adjective and color presentation is printed in the manual accompanying the program package). The data presented are the mean reaction times for each condition.

Optical illusions The purpose of these demonstrations is to reproduce the Müller-Lyer, Ponzo, and Poggendorff illusions and the Phi phenomenon. Given the nature of the computer screen resolution, these programs are not intended for data collection but for illustration of the illusions. For each of the illusions, the specific stimulus pattern is displayed on the screen. The subject may then manipulate the lines until there is apparent equality in length or continuity. Upon subject request, the error, if any, is indicated. For the Phi phenomenon, a pattern of flashing lights is presented. The rate of flashing can be controlled to create the perception of apparent movement.

Movement programming. This demonstration is a reaction time experiment that requires that the subject perform one, two, or three separate operations after a signal is presented. With this method, it may be demonstrated that there is a "programming period" that occurs prior to the execution of a chain of behaviors, and that this programming time increases as the number of operations is increased (see, e.g., Klapp, Wyatt, \& Lingo, 1974). Essentially, each trial begins with instructions to press one, two, or three keys in a sequence after a cue tone is heard. The subject then starts the trial by placing his/her finger on the space bar. After a variable interval of 2$3 \mathrm{sec}$, the tone is sounded, and the reaction times for releasing the space bar and pressing the first key in the sequence are recorded. For all trials, the " $\mathrm{Z}$," " " $\mathrm{A}$," and " $Q$ " keys are used; the three sequences are " $A$," " $Z$ $A$," and "Z-A-Q." The mean reaction times for each of the dependent variables are reported for the three conditions. Significant differences between the times to release the space bar are hypothesized to be a function of the programming period.

Figure detection. This program demonstrates the visual scanning operation discussed by Neisser and Beller (1965). In brief, the subject is presented with an array of letters and is instructed to search for a specific cue letter. The time that the subject requires to locate the cue is a function of the similarity of the cue and the surrounding letters and of the relative position of the cue within the matrix. In general, the search time increases as the cue is presented lower in the matrix and when the cue and the background share similar features. For this demonstration, the independent variables are the cue letter to be found ( $X$ vs. $Q$ ), the background letters [angled letters $(K, M, N, V$, and $W)$ vs. rounded letters $(C, G$, $\mathrm{O}, \mathrm{S}$, and $\mathrm{U})]$ in which the cue is embedded, and the row ( 1 through 12) in which the cue letter is located. On each trial, a matrix of $8 \times 12$ letters is printed in the center of the screen. The matrix is composed of one of the background types and contains a single cue located in one of the 12 rows. The subject is instructed to search through the array, locate the specific cue, and hit any key when the cue is located. The subject's reaction time for finding the cue is measured. Each of the 48 possible independent variable combinations is presented randomly five times during the demonstration. The mean reaction times for each condition (cue $\times$ background $\times$ position) are presented in tabular form at the end of the experiment.

Language utilization. Clark and Chase (1972) posited that both written descriptions and pictures are encoded in the same general fashion, which allows for quick comparisons of the two. Furthermore, there are four specific stages that are used when the two are compared. The algorithms that are used to compare the information received from both a picture and a sentence can be studied, in part, by measuring the time it takes one to determine whether the description and the picture are in agreement. This program is modeled after the first experiment reported by Clark and Chase (1972). On each trial, the subject is presented a sentence-picture pair and is required to determine whether the sentence is true of the picture. The picture consists of either an asterisk above a plus sign or a plus sign above an asterisk. The descriptive sentences are printed in capital letters directly to the left of the picture. These sentences are either positive statements (e.g., "The star is above the plus" or "The star is below the plus") or negative statements (e.g., "The star is not below the plus" or "The star is not above the plus"), with the words "plus" and "star" being interchangeable. For all sentence-picture pairs, half are true statements and half are false. On each trial, the subject must use the " $<$ ", and the " $>$ " keys to indicate whether the sentence is true of the picture. The data collected are the number of correct responses to the stimulus configuration and the mean reaction time for the correct responses.

Visual and name coding. Posner, Boies, Eichelman, and Taylor (1969) demonstrated that the visual code for a letter (case and figure) is more quickly processed than in the symbolic code (name of the letter). The demonstration noted here is a reconstruction of the third experiment reported by Posner et al. During the first part of the demonstration, a capital letter is presented for $0.5 \mathrm{sec}$. After an interstimulus interval of $0-1 \mathrm{sec}$, a second capital letter is presented, and the subject must indicate whether the two letters are the same or different by pressing the " <" key or the " > " key, respectively. The reaction times of correct responses are recorded; incorrect responses are ignored. The letters are the same as those used by Posner et al. (A, B, F, H, and K), as are the interstimulus intervals $(0.0,0.5$, and $1.0 \mathrm{sec})$.

During the second phase of the demonstration, the first letter presented is capitalized, whereas the second letter may be printed in either upper- or lowercase. Again, the subject's reaction time for determining whether the letters are the same or different is recorded. 
High-speed memory scanning. This program is based on the experiments reported by Sternberg (1966) concerning the rate at which short-term memory is scanned. In essence, the subject memorizes a list, or memory set, containing two, three, or six digits. Once the set has been memorized, the subject is shown single-digit numbers and is required to determine whether or not the stimulus number is a member of the memory set. For this program, there are three phases representing each of the memory set sizes. Because the "fixed-set" (Sternberg, 1966) procedure is used, during each phase a set of digits is generated randomly by the program and is presented to the subject for memorization. Once the subject has demonstrated that he/she has memorized the list, the memory scanning process is examined by measuring the subject's reaction time for determining whether the stimulus is a member of the memorized list.

Short-term-memory forgetting. Peterson and Peterson (1959) demonstrated that most information stored in short-term memory is lost within $18 \mathrm{sec}$. The method requires that the subject be exposed to a three-letter trigram for $2 \mathrm{sec}$. Immediately after the $2 \mathrm{sec}$ has elapsed, the subject is engaged in a masking task for a specified length of time, after which he/she is required to recall the memorized trigram. On each trial, this program randomly generates a trigram and presents it on the screen for $2 \mathrm{sec}$. The masking task requires that the subject determine whether a series of numbers are greater than or less than 500 . The intervals between the end of the memorization period and the recall are $0,2,4,6,10$, and $18 \mathrm{sec}$. After the masking trial, the subject types in the trigram that is remembered. To determine whether the subject has entered the same trigram, this trigram is then compared with the one generated by the computer.

Secondary task performance. Britton and Tesser (1982) suggested that the cognitive capacity of an individual varies as a function of prior knowledge. Specifically, high prior knowledge increases the cognitive capacity used in the situation. This change in cognitive capacity can be evaluated by observing a subject's performance on a secondary task, such as a simple reaction time task. In general, increases in prior knowledge appear to create a commensurate decrease in efficiency on the secondary task performance as measured by reaction times. This effect can be demonstrated by having subjects read a passage of text, at a fixed rate, with or without prior information about the subject of the text, and having them make a timed reaction to a randomly presented tone. This program allows for a long text to be broken up into a series of chunks of a specified length, with each chunk presented sequentially on the screen for a fixed time. During the presentation of different chunks, a tone can be sounded to indicate that the subject should press the " > " key. Included within the package are three support programs that allow for creating a sequence file in file in which text is stored, editing of the text for placement of the program controls, and creating the chunked text file. We have included several chunked text files on the disk for use with this demonstration.

Concept formation. The process of concept formation entails the development of rules or propositions that allow for the organization and categorization of relevant objects and events into meaningful groups. One factor that affects the time required to form a concept is the complexity of the concept. In this demonstration, two conceptual rules are tested, affirmation and conditional. The two concepts are tested separately in different phases of the demonstration, and the number of trials required to reach a criterion of 10 successively correct responses is recorded for each concept. For both concepts, the task is essentially the same. On each trial, the subject is shown a trigram composed of graphic characters (asterisk, club, diamond, heart, pound sign, and spade) and is required to determine whether the trigram is "true" or "false." For the affirmation concept, the spade must be present in the trigram in order to be a "true" statement. For the conditional task, when the asterisk is printed on the right side of the trigram, the club must be present to be considered "true"; all other possibilities are considered "false." Using the " $<$ " or the " $>$ " keys, the subject indicates whether the trigram is "true" or "false," respectively, and is given immediate feedback on the accuracy of the response. If the subject fails to respond within $4 \mathrm{sec}$, the trial ends and the next trial begins.

Opponent process - colors. With this program, the opponent process theory of color vision can be demonstrated. (This program can be used only with the Commodore 64.) On each trial, a large colored square is presented on the screen for $3 \mathrm{sec}$. The square appears in the center of the screen and is $12 \times 18$ character blocks in size. Each block is the individual unit of screen space, used by the computer for the display of graphics-the colored square covers approximately $20 \%$ of the screen. There is a fixation point printed in the center of the screen, and the background is gray. After the $\mathbf{3 ~ s e c}$, the screen is cleared, leaving the gray background, and an afterimage is typically perceived. The colors used are red, cyan, purple, green, blue, yellow, orange, and black. There is a 3-sec interstimulus interval between trials.

Correlation program. The correlation program is a small statistical package that allows the user to enter data for analysis with both simple correlation and linear regression. Any number of variables can be entered. The only requirement is that there be an equal number of observations for all variables. The statistics provided for each variable are the mean, standard deviation, correlations for all possible combinations, and summation values (e.g., sum of cross products for each variable combination). Student $t$ tests are conducted for each reported correlation. The user can then choose to perform a simple linear regression or any combination of variables and can assign the appropriate variables as being either the independent or the dependent variable. The linear equation is printed, and the $\mathrm{F}$ ratio for the regression is reported. The 
program also allows for storage of the data on disk for later analysis or modification.

\section{Availability of Programs}

A complete listing of the programs in the package, a copy of the current manual, and the programs on disk may be obtained for the cost of reproducing and mailing, estimated to be $\$ 10.00$. The disk is formatted for operation on the Commodore 4040, 2031, and 1541 disk drives and other non-Commodore drives that are compatible with the Commodore system.

\section{REFERENCES}

Brain, D. A., Oviate, P. R., Paquin, P. J. A., \& Stone, C. D. (1981). Basic conversion handbook: For Apple, TRS-80, and Pet users. New York: Hayden.

Britton, B. K., \& Tesser, A. (1982). Effects of prior knowledge on use of cognitive capacity in three complex cognitive tasks. Journal of Verbal Learning and Verbal Behavior, 21, 421-436.
Clark, H. M., \& Chase, W. G. (1972). On the process of comparing sentences against pictures. Cognitive Psychology, 3, 472-517.

GoRDON, C. J. (1974). Effect of differing number of colors on the Stroop Color and Word Test. Perceptual and Motor Skills, 39, 1067-1070.

KlapP, S. T., Wyatt, E. P., Lingo, W. M. (1974). Response programming in simple and choice reactions. Journal of Motor Behavior, 6, 263-271.

NeISSER, U., \& Beller, H. K. (1965). Searching through word lists. British Journal of Psychology, 56, 349-358.

Peterson, L. R., \& Peterson, M. J. (1959). Short term retention of individual verbal items. Journal of Experimental Psychology, 58, 193-198.

Posner, M. L., Boies, S. J., Eichelman, W. H., \& Taylor, R. L. (1969). Retention of visual and name codes of single letters. Journal of Experimental Psychology Monographs, 79 (1, Pt. 2).

SIMPSON, G. B., \& BURGESs, C. (1984). PET programs for demonstration and student research in cognitive psychology. Behavior Research Methods, Instruments, \& Computers, 16, 65-66.

STERNBERG, S. (1966). High-speed scanning in human memory. Science, $153,652-654$.

(Revision accepted for publication February 26, 1985.) 